\title{
Design and Analysis of a Toroidal Tester for the Measurement of Core Losses under Axial Compressive Stress
}

\author{
Natheer Alatawneh ${ }^{1}$, Member, IEEE, Tanvir Rahman ${ }^{2}$, David A. Lowther ${ }^{2}$, and Richard Chromik ${ }^{1}$ \\ ${ }^{1}$ Department of Mining and Materials Engineering, McGill University, QC, Canada, H3A 0G4 \\ ${ }^{2}$ Department of Electrical and Computer Engineering, McGill University, QC, Canada, H3A 0E9
}

\begin{abstract}
Electric machine cores are subjected to mechanical stresses due to manufacturing processes. These stresses include radial, circumferential and axial components that may have significant influences on the magnetic properties of the electrical steel and hence, on the output and efficiencies of electrical machines. Previously, most studies of iron losses due to mechanical stress have considered only radial and circumferential components. In this work, an improved toroidal tester has been designed and developed to measure the core losses and the magnetic properties of electrical steel under a compressive axial stress. The shape of the toroidal ring has been verified using 3D stress analysis. Also, 3D electromagnetic simulations show a uniform flux density distribution in the specimen with a variation of $0.03 \mathrm{~T}$ and a maximum average induction level of $1.5 \mathrm{~T}$. The developed design has been prototyped, and measurements were carried out using a steel sample of grade 35WW300. Measurements show that applying small mechanical stresses normal to the sample thickness rises the delivered core losses, then the losses decrease continuously as the stress increases. However, the drop in core losses at high stresses does not go lower than the free-stress condition. Physical explanations for the observed trend of core losses as a function of stress are provided based on core loss separation to the hysteresis and eddy current loss components. The experimental results show that the effect of axial compressive stress on magnetic properties of electrical steel at high level of inductions becomes less pronounced.
\end{abstract}

Index Terms - Electrical Steel, Compressive Axial Stress, Core Losses, Hysteresis Loss, Eddy Current Loss, Magnetic Properties, Toroidal Tester, and FEM analysis.

\section{INTRODUCTION}

I RON loss calculations in electrical machines are important for determining the efficiency of electromagnetic devices. Often, there are significant discrepancies between the predicted and measured values of the efficiency. One reason for the discrepancy between simulation results and measurements could be due to the omission of effects from manufacturing processes such as shrink fitting, cutting, pressing and clamping of steel laminations [1-5]. Shrink fitting, in particular, can have a significant impact [6]. Shrink fitting is a manufacturing technique by which metal components are combined together. Application of shrink fitting for an electric machine includes the shaft-rotor and the stator-house assembly processes, among others. This method of assembly is widely used in the industry and leaves residual mechanical stresses on the machine components which can affect the magnetic properties of the electrical steel and thus the electromechanical behavior of the machine [7-9].

Structural analysis of an electrical machine assembled by shrink fitting shows that the machine components experience three types of stresses including radial, hoop (circumferential) and axial stresses. Due to the lack of experimental data machine designers and finite element (FE) software packages consider only the effect of radial and hoop stresses during core loss calculations [10-12], i.e., the effects of axial stress are generally ignored. Previously, a few articles have reported magnetic measurements including the B-H properties, hysteresis loops, core losses etc. of electrical steel under axial compressive stress [13-16]. However, the homogeneity of the stress distribution within the sample of the experimental fixture (an important requirement for such measurements) was not investigated and previous studies have used a superficial measure of the stress using the lamination sample surface area and the load (assuming uniform stress distribution), which is dependent on the apparatus and is not necessarily applicable.
In this paper, an improved design test fixture has been developed for the measurement of the magnetic properties of electrical steel laminations under mechanical stress in the axial direction. The design process involved a study of stress distribution to choose the proper material of the frame and calculate the applied stress on the sample accurately. The originality of this work can be seen from different angles; First: The improved design offers a better stress distribution compared with prior designs. Second: A FE study on the structural mechanics of the test fixture has been performed for the first time. This analysis helps in exploration the stress distribution within the sample, in addition, it provides a solid guideline to choose the proper material of the fixture to tolerate the desired applied stress. Third: A compensation coil has been developed which is basically a replication of the fixture without the sample. In literature, the toroid for compressive stress measurements was used without compensation coil [17], or an approximated mathematical factor was implemented to compensate for the flux leakage [18]. Fourth: Extensive measurements have been executed for a stress range up to $32.3 \mathrm{MPa}$ (where in literature the maximum possible stress was $28 \mathrm{MPa}$ [17]), with various frequencies from $50 \mathrm{~Hz}$ to $1 \mathrm{kHz}$. Also, physical interpretation for the loss trend as a function of stress is provided.

The remainder of this paper is organized as follows. Section II describes the proposed ring tester design. 3D FE analyses of structural mechanics and electromagnetics are carried out in sections III and IV respectively. A detailed description of the experimental setup is presented in section $\mathrm{V}$, which is followed in Section VI by results and discussion. Finally, section VII provides some final conclusions and directions for future work.

\section{RING TESTER DESIGN}

The determination of the magnetic properties of lamination 
materials as a function of stress requires setting up an experimental apparatus with a closed magnetic circuit in which the test samples are exposed to uniform in-situ stress distributions. To satisfy this condition a toroidal tester has been developed. The tester is composed of a single ring steel sample with inner and outer diameters of 60 and $80 \mathrm{~mm}$, respectively (the reasons for choosing these dimensions are due to the dimension of the mechanical hydraulic press and magnetics related considerations discussed below). The sample is set in between two insulator rings of the same diameters as the sample. Each insulator ring has a thickness of $10 \mathrm{~mm}$ with 12 semi-circular grooves drilled from the side around the insulator perimeter. Each groove has a diameter of $8 \mathrm{~mm}$, as shown in Fig. 1. The excitation and measuring coils are wrapped in the grooves which allows the axial force to be applied directly to the insulators and transferred to the specimen. The insulator rings are made from cast acrylic [Poly (methyl methacrylate)], which is a dielectric material $\left(\mu_{\mathrm{r}}\right.$ slightly less than 1) that allows the generated flux inside the winding to flow within the specimen. Other properties which encourage the use of acrylic are: its ability to withstand high temperature (recommended continuous service temperature $85^{\circ} \mathrm{C}$ ), cost effectiveness, high coefficient of expansivity, durability and ease of assembly [19, 20].

Because of the fact that the magnetic field in the sample is non-uniform (larger near the inner perimeter and lower at the external edge), the sample dimensions have been chosen to fulfil the condition of $D_{\mathrm{o}} / D_{i}<1.6$, where $D_{\mathrm{o}}$ and $D_{i}$ are the inner and the outer diameters, respectively [21]. When the ration $D_{\mathrm{o}} / D_{i}$ is less than 1.1 the length of magnetic path is $2 \pi r_{\mathrm{o}}$, where $r_{\mathrm{o}}$ is the average of inner and outer radii. However, if the ratio is greater than 1.1, the length of magnetic path has to be recalculated to compensate for the non-homogeneity of the flux. For the range of $1.1<D_{\mathrm{o}} / D_{i}<1.6$ (as in our case $D_{\mathrm{o}} / D_{i}=$ 1.33 ) the mean length of magnetic path is given by,

$$
l_{m}=\pi \frac{D_{o}-D_{i}}{\ln \left(D_{o} / D_{i}\right)}
$$

In general, the higher the $D_{\mathrm{o}} / D_{i}$ ratio, the lower the uniformity of flux within the sample, thus the reliability of measurements is lower. However, the measurements can be improved since a larger $D_{\mathrm{o}} / D_{i}$ means a larger volumetric size of the sample and hence, the influence of the leakage flux in the air gap (between the coil and the sample) is less pronounced. Also, a larger ratio offers a larger sample surface that can be subjected to stresses, and consequently the influence of stress is more visible. To compromise between the prototyping effort, flux uniformity distribution and visibility of stress effect, a ratio of 1.33 was chosen.

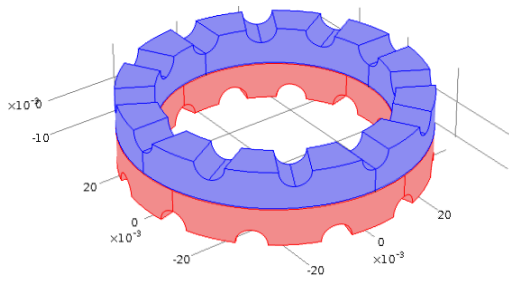

Fig. 1: The insulator rings with semi-circular side grooves

\section{Structural Mechanical ANALYsis}

One of the goals of designing an appropriate tester is to ensure that the stress distribution in the sample is uniform. This can be investigated by performing a mechanical structural analysis of the apparatus. We carried out a number of simulations to determine the optimal shape of the insulator frame including the effect of different groove shapes (semicircular and square), and the relative groove alignments (staggered and non-staggered) between the upper and lower insulators. These two aspects of the design affect the uniformity of the resulting stress distribution the most. Fig. 2 shows the initial design of the insulator rings with aligned square and semi-circular grooves. The material properties of the rings have been provided in Table I. When a force of $35 \mathrm{kN}$ is applied perpendicularly over the frame, the results of finite element analysis of the problem are shown in Figs. 3-6. A parameter which has been frequently used to assess the safety of the material against deformation is the Von Mises stress distribution in the material samples. In Figs. 3 and 4 the maximum value of the Von Mises stress induced in the material of the insulator frame (112.9 MPa for square and 108 MPa for semi-circular) is less than the strength of the material (124 MPa for cast acrylic). This means that the material would not deform at this applied pressure. Also, the semi-circular groove design achieves a lower maximum Von Mises stress compared with the square design for the same applied force. One more advantage of using the semi-circular groove design can be observed from the results of z-axis stress distribution on the test sample as shown in Figs. 5 and 6. The mechanical stress is distributed more uniformly over the sample in the semi-circular design with a variance of $4.43 \times 10^{13}$ compared to $5.59 \times 10^{13}$ for the square design. The variance decreases by 20\%. Another design improvement can be achieved by rotating the upper insulator in order to obtain staggered groove alignment between the upper and lower insulator rings, as shown in Fig. 1. A stress analysis comparison between the staggered and the aligned semi-circular groove design has been performed and the simulation results are presented in Figs. 7 and 8. The maximum Von Mises stress in the insulator ring drops to $98.6 \mathrm{MPa}$ compared to $108 \mathrm{MPa}$ for the aligned design. This means that higher forces can be applied to the staggered apparatus. Moreover, the variance of the stress distribution over the specimen decreases by $\sim 45 \%\left(2.37 \times 10^{13}\right.$ compared to $4.43 \times 10^{13}$ in the aligned design). The all over improvement in the stress variation within the sample from the square design used in literature to the current proposed design is almost $57.6 \%$. It is worthwhile to point out that the average of the stress over the sample surface calculated from the FE result increases for the same applied force; 15.69 MPa, 15.76 $\mathrm{MPa}$ and 16.0 MPa for the square groove design, aligned semi-circular groove design and staggered semi-circular groove design, respectively. In practice, the sample surface irregularities may cause minor non-axial stresses which are neglected here. 


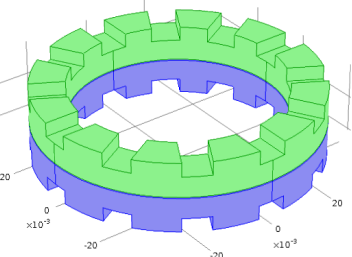

(a)

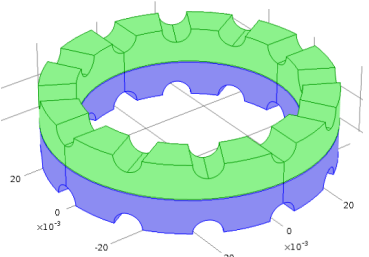

(b)
Fig. 2: The insulator rings with aligned (a) square grooves (b) semi-circular grooves

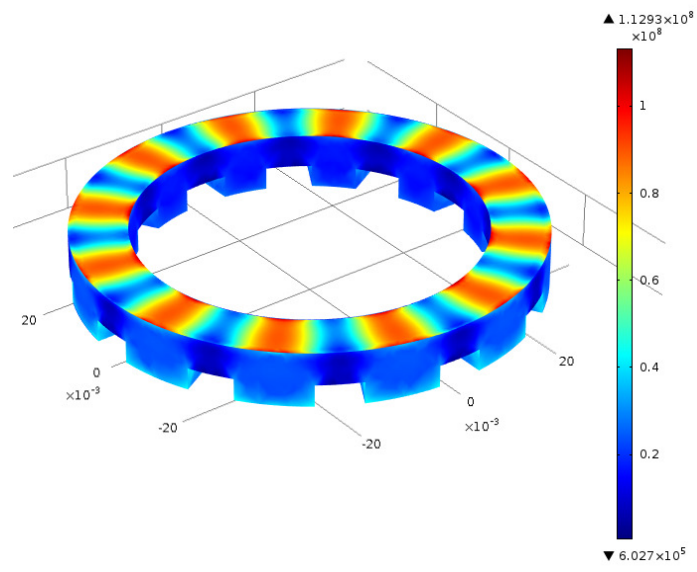

Fig. 3: Von Mises stress distribution $\left[\mathrm{N} / \mathrm{m}^{2}\right]$ over the lower insulator of the square groove design

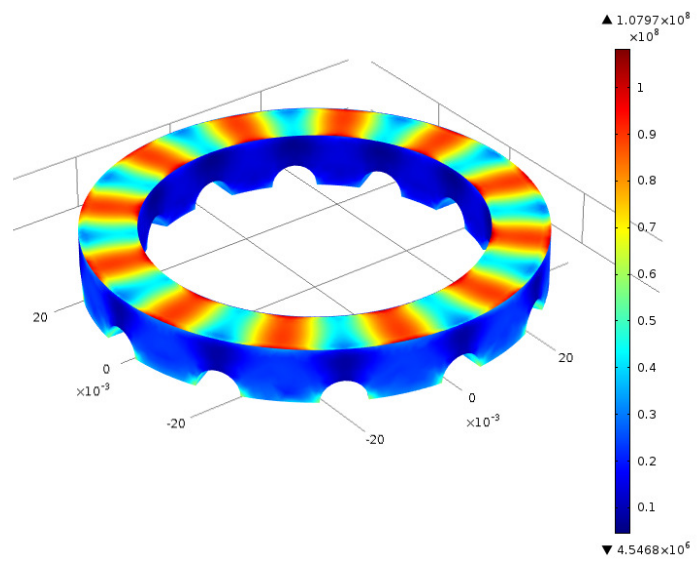

Fig. 4: Von Mises stress distribution $\left[\mathrm{N} / \mathrm{m}^{2}\right]$ over the lower insulator of the semi-circular groove design

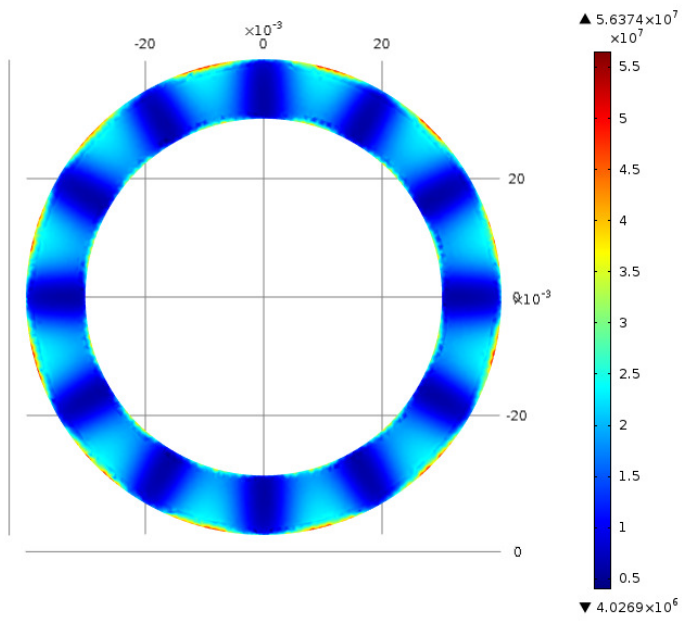

Fig. 5: The stress distribution $\left[\mathrm{N} / \mathrm{m}^{2}\right]$ on the z-direction over the specimen in the square groove design

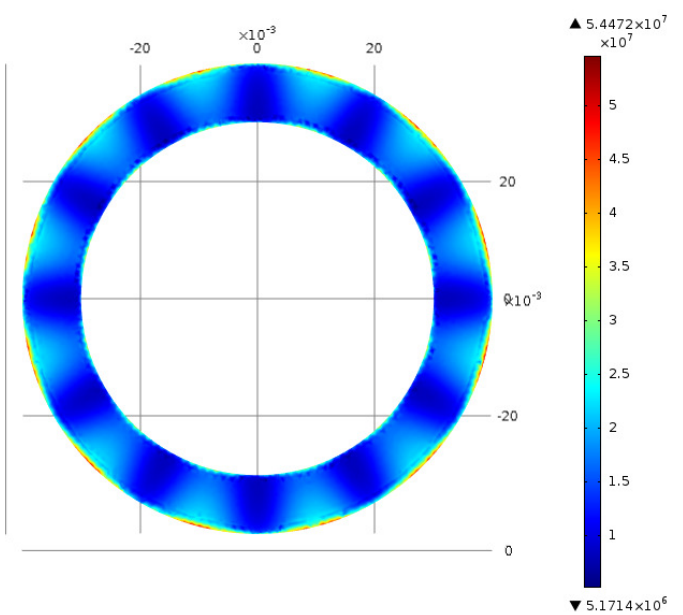

Fig. 6: The stress distribution $\left[\mathrm{N} / \mathrm{m}^{2}\right]$ on the z-direction over the specimen in the semi-circular groove design

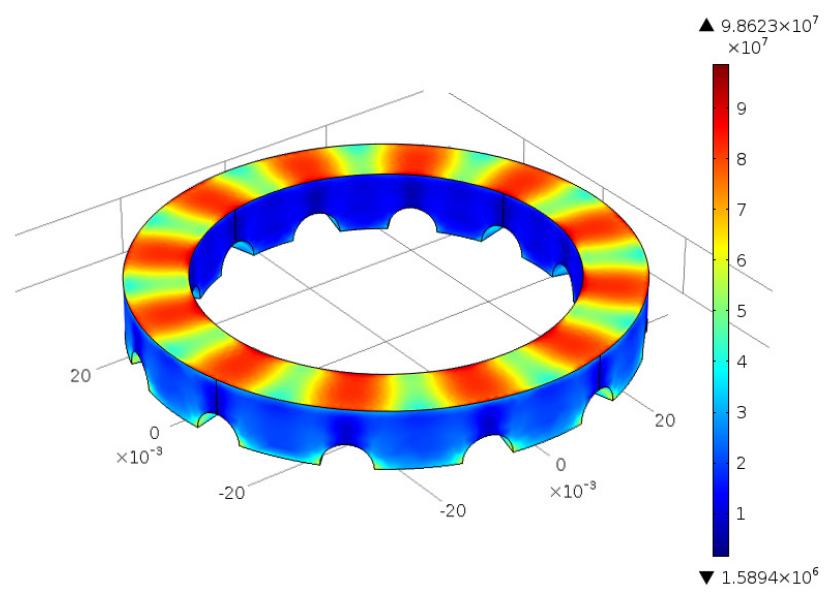

Fig. 7: Von Mises stress distribution $\left[\mathrm{N} / \mathrm{m}^{2}\right]$ over the lower insulator of the staggered semi-circular groove design 


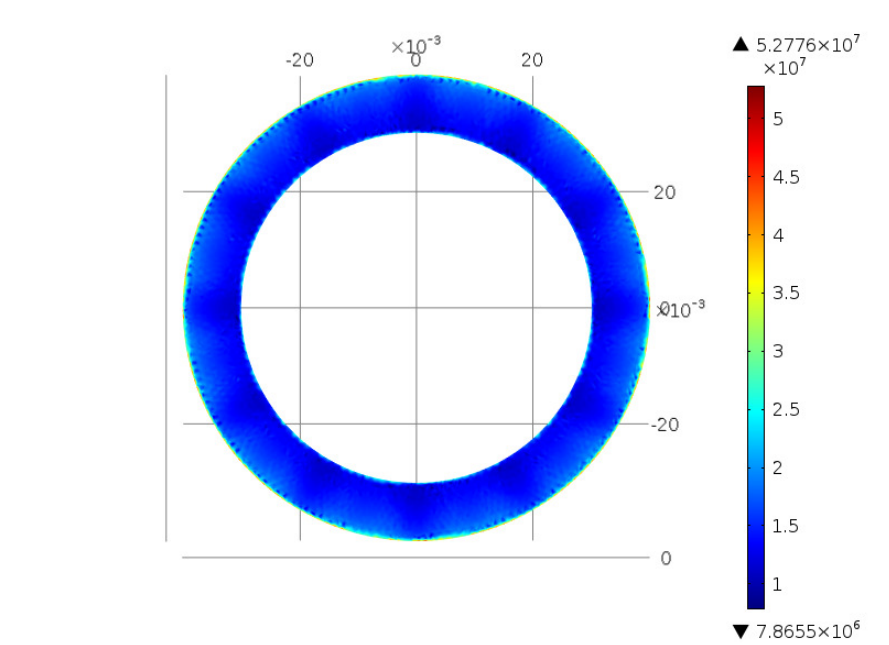

Fig. 8: The stress distribution $\left[\mathrm{N} / \mathrm{m}^{2}\right]$ on the z-direction over the specimen in the staggered semi-circular groove design

TABLE I

THE MATERIAL PROPERTIES

\begin{tabular}{lcc}
\hline \hline & Cast Acrylic & Electrical Steel \\
\hline \hline Young's modulus [G Pa] & 3.2 & 185 \\
\hline Poisson's ratio & 0.35 & 0.29 \\
\hline Density $\left[\mathrm{kg} / \mathrm{m}^{3}\right]$ & 1190 & 7650 \\
\hline
\end{tabular}

\section{ELECTROMAGNETIC ANALYSIS}

Besides carrying out mechanical stress analysis to obtain an optimal shape of the apparatus another important analysis to be done is the electromagnetic simulation of the setup to study the electromagnetic field distribution in the sample. The electromagnetic finite element analysis of the problem has been carried out in 3D. The geometry of the toroid is shown in Fig. 9 (a). Before presenting the results of the electromagnetic analysis a summary of the apparatus is given. Helical windings have been used due to the use of staggered grooves. Each groove accommodates 24 conductors of gauge 24 (AWG) copper wire. In total, the primary winding consists of 288 turns (288 conductors was selected to ensure that the apparatus could drive our material sample into saturation $(\sim 1.6$ $\mathrm{T}$ ) at frequencies up to $1 \mathrm{kHz}$.). The simulation result of the flux distributions within the sample is shown in Fig. 9 (b), when a current of $1 \mathrm{~A}$ is imposed in the primary windings. Results show a homogeneous flux distribution with a variation of almost $0.03 \mathrm{~T}$ between the inner and external edges of the sample. The average value of the flux density is about $1.5 \mathrm{~T}$.

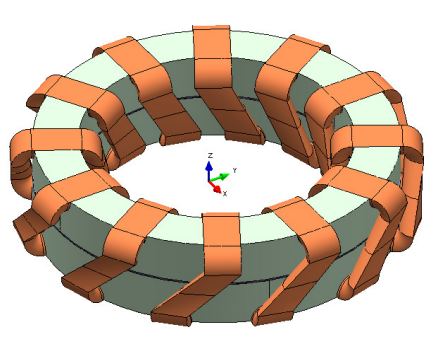

(a)

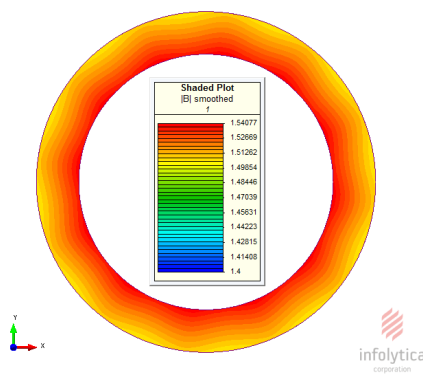

(b)

\section{EXPERIMENTAL SET-UP}

The proposed toroid is built using 288 turns of primary winding, and a pick up coil of 120 turns for the measurement of magnetic flux density $\boldsymbol{B}$. The magnetic field strength $(\boldsymbol{H})$ can be calculated using Ampere's law,

$$
H=\frac{N . i}{l_{m}}
$$

The mean length of the magnetic path $l_{m}$ is calculated by (1) and is equal $218.41 \mathrm{~mm}$. The developed toroid is shown in Fig. 10 (a). A specimen of electrical steel of grade 35WW300 has been used. The specimen was prepared using laser cut to decrease the effect of cutting to the minimum, and then an annealing heat treatment was performed at a temperature of $700^{\circ} \mathrm{C}$ for two hours to relieve thermal stresses induced by the laser cut process. The toroid is linked to the ring tester in a commercial magnetic measurement system, which provides robust measurements and reproducibility better than $0.2 \%$. A manual hydraulic press is employed to apply the stress on the sample. The measurement system including the toroid inside the press is shown in Fig. 10 (b). Since the cross section of the secondary coil is fairly greater than the cross section of the sample, the air flux contributes to the measured induced voltage by the coil. Thus, the calculated flux density $\boldsymbol{B}$ from the measured secondary coil would be larger than $\boldsymbol{B}$ in the specimen. Therefore, it is necessary to compensate for the air flux. In Epestine frame, the air flux compensation is performed using a mutual inductance $M$ wound on a nonmagnetic cylindrical former placed at the middle of the Epestine frame. The primary winding of $M$ is connected in series with the primary winding of the frame, while the secondary of $M$ is connected in series opposition to the secondary of the frame. The mutual inductance can be adjusted in such a way that the induced voltage from both secondary coils in series is zero in the absence of the specimen. This adjustment method is not applicable in toroid where the specimen cannot be removed while keeping the winding structure. Therefore, the air flux compensation for this developed design is accomplished by replicating the same toroidal system without the specimen. Fig. 11 shows both toroids. The measured hysteresis loops at a peak of $1.5 \mathrm{~T}$ before and after compensation are shown in Fig. 12. It is clearly observed that for the same magnetic field strength $\boldsymbol{H}$, the $\boldsymbol{B}$ is overestimated without the air flux compensation.

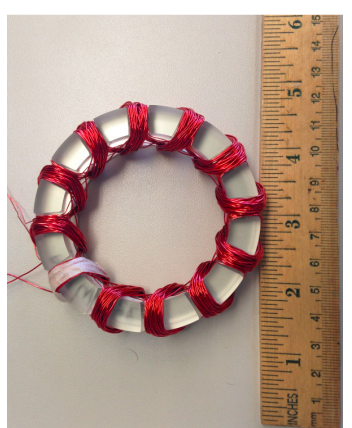

(a)

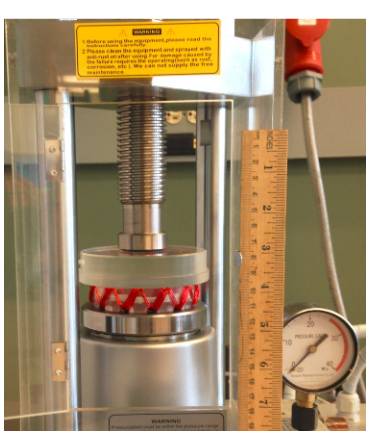

(b)
Fig. 10: (a) Photograph of the developed toroid (b) The measurement system

Fig. 9: (a) The toroid geometry (b) Flux density distribution in the specimen 


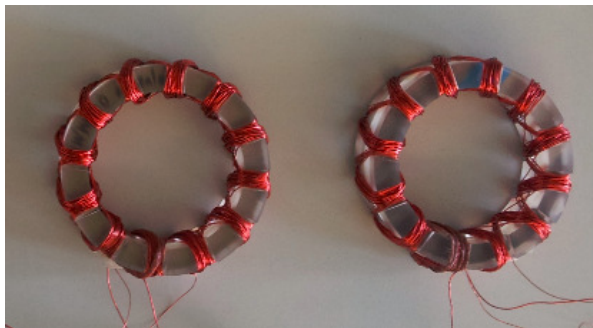

(a)

(b)

Fig. 11: (a) The toroid with an annealed electrical steel sample 35WW300 (b) The compensation inductance

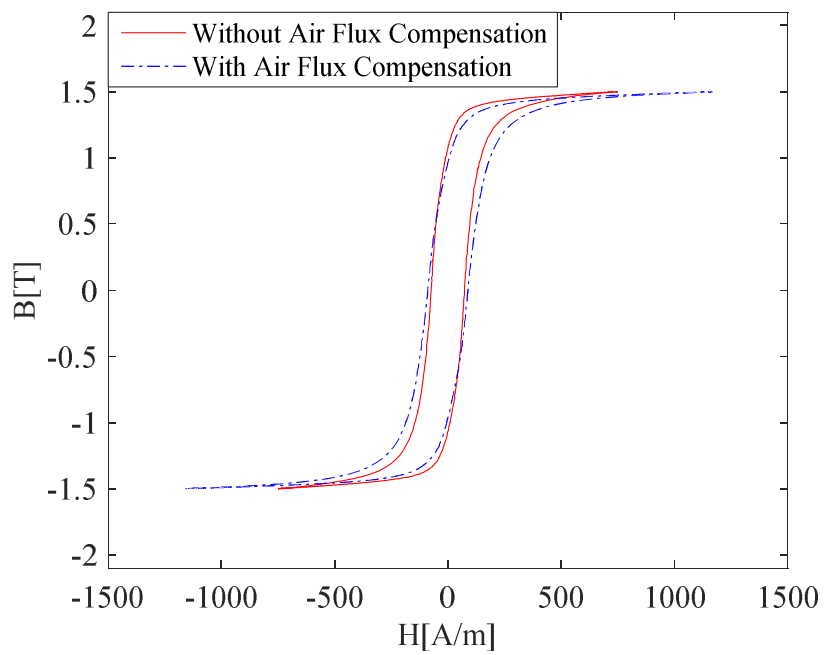

Fig. 12: Measured hysteresis loops obtained from the measurements of an annealed electrical steel sample $35 \mathrm{WW} 300$ at $50 \mathrm{~Hz}$ with and without compensation

\section{RESULTS AND DISCUSSION}

Measurements were carried out on a specimen of grade 35WW300 under different stresses, flux densities, and excitation frequencies. Fig. 13 presents core losses in the specimen under a sinusoidal excitation with a frequency of 50 $\mathrm{Hz}$ at three levels of flux density, 0.5, 1.0, and 1.5 T. The figure suggests that core losses increase in the beginning when a small load is applied, then the losses decrease continuously as the stress increases. This trend can be explained physically in terms of the fact that a small compressive stress applied normal to the sample surface impedes the movement of the domains while the domains try to align themselves with an external magnetic field, thus, more energy loss is dissipated during the magnetization process. When the applied stress increases the sample shows a fairly stretch in the $x-y$ plane. This is equivalent to the situation of exposing the sample ring to tensile stresses in radial and circumferential directions. It is well known in literature that the tensile stress causes domain refinement, where domains are divided into narrower ones and domain wall spacing decreases, therefore, core loss decreases $[18,21,22]$. With a higher frequency of $1 \mathrm{kHz}$, the delivered core losses in the sample show the same trend of increase in losses at small applied stresses and then decrease in losses with the increase of stresses, as illustrated in Fig. 14. This proves that the observed trend is not only related to hysteresis losses but also to eddy current losses, where at high frequency the eddy current loss component is objectively dominant. The losses at high and low frequencies $(50 \mathrm{~Hz}$ and $1 \mathrm{kHz}$, respectively) are separated to hysteresis and eddy current loss components as depicted in Figs. 15 and 16. The loss separation has been accomplished based on the extrapolation method by using a range of frequencies of 50, 100, 200, 400, 600,800 , and $1000 \mathrm{~Hz}$. A detailed description of the loss separation method under stresses can be found in [23]. Results are verified by showing that the hysteresis loss component is more pronounced compared with eddy loss component at $50 \mathrm{~Hz}$, the low frequency. While the eddy current loss component is dominant at $1 \mathrm{kHz}$, the high frequency. It is clearly shown in Figs. 15 and 16 that both loss components follow the trend of increasing and then decreasing along the stress profile. In Fig. 16, it is clearly shown that the eddy current loss increases when the sample is subjected to a small axial stress. This increase can be explained as follows: When the specimen is magnetized at a given flux density, the specimen is expanded by a displacement $\lambda$ owing to the magnetostriction phenomenon. Once the displacement happens under compressive stress, an extra magnetic work is produced to resist the external mechanical stress [24]. Recall that the domain rearrangement process enhances eddy current losses and consequent heating around the moving walls [25, 26]. As the compressive stress increases the magnetic loss increases until a certain stress causes stretching in $x-y$ plane. At that certain compressive stress the specimen thickness starts reducing with the increase of the stress, and it is commonly known that eddy current loss decreases as the specimen thickness decreases [27].

An important property that characterizes the magnetic material, rather than the delivered core loss, is the relative permeability, $\mu_{\mathrm{r}}$. The influence of the axial compressive stress on the relative permeability under various stresses is shown in Fig. 17. Each permeability profile was measured for the same magnetic field strength $(\boldsymbol{H})$ as a function of applied compressive stress, s.t. $\mu_{\mathrm{r}}(\boldsymbol{H}, \sigma)$. The relation was chosen to be represented at a constant $\boldsymbol{H}$ in preference of $\boldsymbol{B}$ because the range of $\boldsymbol{H}$ is quite larger than the range of $\boldsymbol{B}$, thus clearer profiles can be shown to track the change in permeability. Fig. 17 shows the variation of $\mu_{\mathrm{r}}$ as a function of stress at four different levels of induction $\boldsymbol{H}$ with $50 \mathrm{~Hz}$ frequency. The first observation is that at low induction level $\boldsymbol{H}=100 \mathrm{~A} / \mathrm{m}, \mu_{\mathrm{r}}$ decreases until $8 \mathrm{MPa}$, then increases as the stress increases. The same trend can be observed at higher levels $\boldsymbol{H}=300$ and $500 \mathrm{~A} / \mathrm{m}$, but the increase after the $8 \mathrm{MPa}$ becomes less pronounced as $\boldsymbol{H}$ increases. The second observation is related to the high level of induction $\boldsymbol{H}=1000 \mathrm{~A} / \mathrm{m}$, where it is clearly shown that the permeability is almost constant and independent of stress after $8 \mathrm{MPa}$. This can be attributed to the saturation, where the mechanical stress effect becomes less pronounced. Third, and the most importantly, the maximum permeability can be recognized at the absence of stress along any permeability curve. The maximum $\mu_{\mathrm{r}}$ at the free-stress condition means that the compressive stress normal to the sheet thickness deteriorates the magnetic material under any value of stress. 


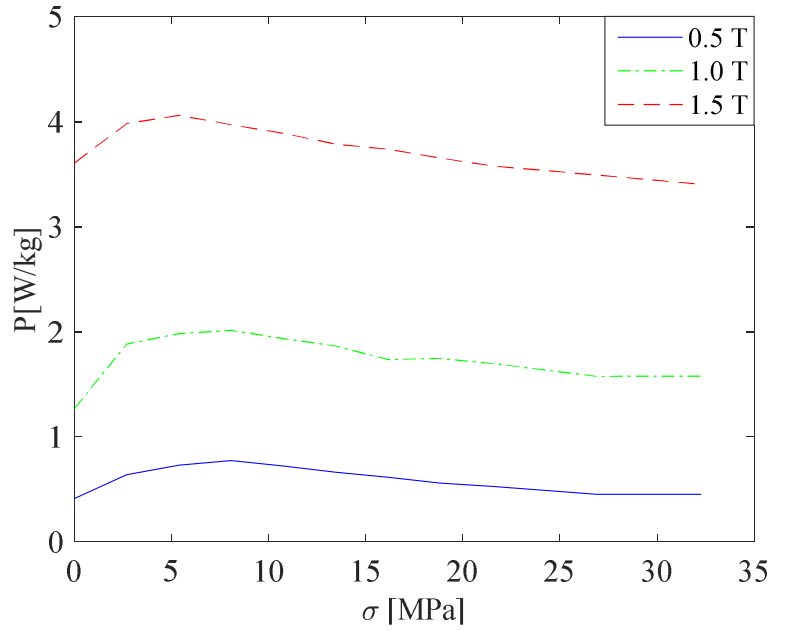

Fig. 13: The measured core losses in 35WW300 electrical steel with different stresses at $0.5,1$, and $1.5 \mathrm{~T}$. The excitation frequency is $50 \mathrm{~Hz}$

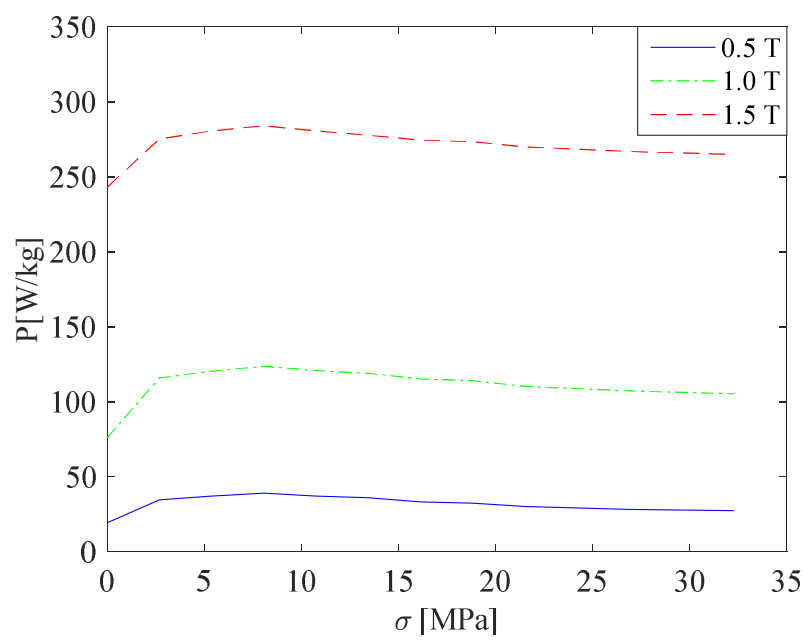

Fig. 14: The measured core losses in $35 \mathrm{WW} 300$ electrical steel with different stresses at $0.5,1$, and $1.5 \mathrm{~T}$. The excitation frequency is $1 \mathrm{kHz}$

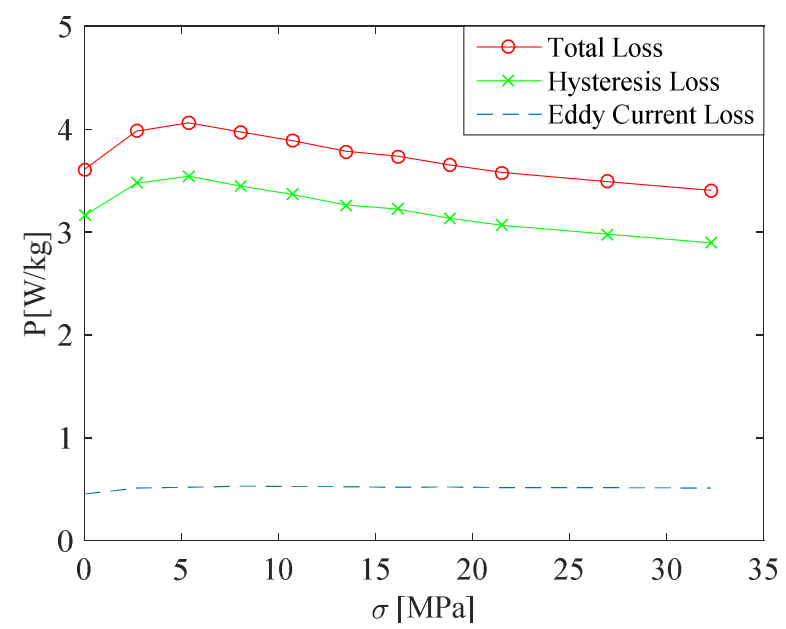

Fig. 15: Loss separation to hysteresis and eddy current components in 35WW300 electrical steel sample with different stresses $1.5 \mathrm{~T}$ and an excitation frequency of $50 \mathrm{~Hz}$

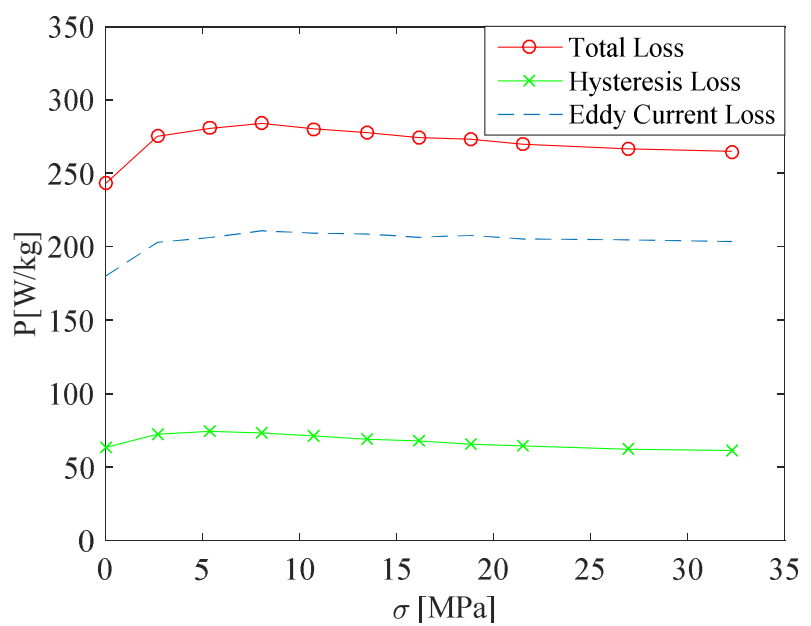

Fig. 16: Loss separation to hysteresis and eddy current components in 35WW300 electrical steel sample with different stresses $1.5 \mathrm{~T}$ and an excitation frequency of $1 \mathrm{kHz}$

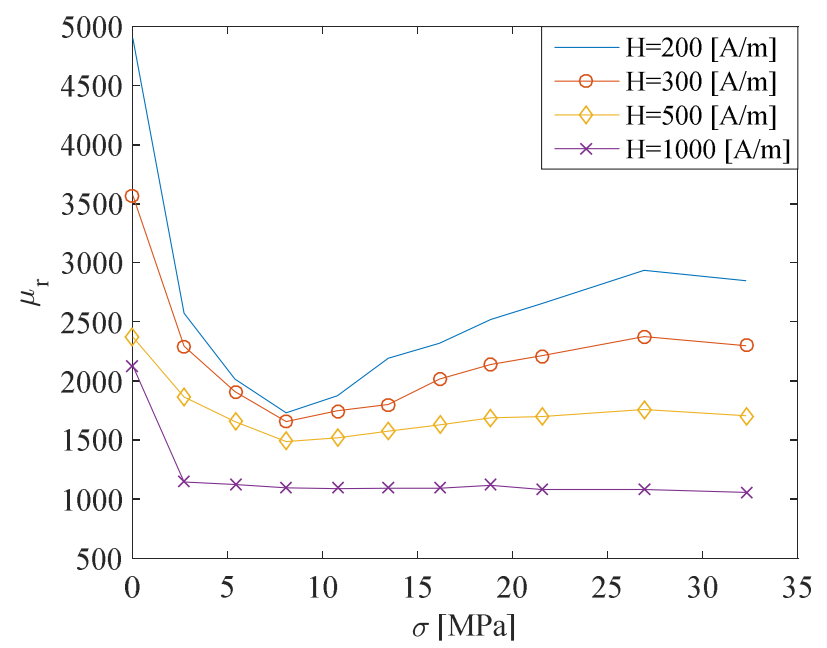

Fig. 17: The relative permeability of the electrical steel sample $35 \mathrm{WW} 300$ as a function of compressive stress with an excitation frequency of $50 \mathrm{~Hz}$

\section{CONCLUSION}

In this paper an improved design of a toroid tester for the measurement of core losses under axial compressive stress has been presented. The proposed design is characterized by semicircular staggered grooves which produce a more uniform and homogeneous distribution of stresses over the specimen. 3D FE analysis on the structural mechanics of the developed toroid can provide a solid guideline to choose the proper material of the fixture to tolerate the desired applied stress. In addition, the stress variation can be investigated in the specimen. Simulation results show that the proposed design may decrease the variation in stress within the sample by almost $57.6 \%$ compared to the square design used in literature. The toroidal tester is compatible with a commercial system which allows robustness, accuracy and decent reproducibility in measurements. The obtained set of experimental results using electrical steel of grade 35WW300 shows that the compressive stress applied normal to the 
specimen thickness deteriorates the magnetic properties of the material at any value of stress. Nevertheless, the core loss profile as a function of stress has an interesting trend where the loss increases at small stresses and then decreases continuously as the stress increases. Also, it was observed that the effect of the axial compressive stress becomes less pronounced at saturation. Future research trends will focus on two streams. Mathematical modeling of core losses as a function of axial compressive stress, and considering the axial compressive stress in the electrical machine design process.

\section{ACKNOWLEDGMENT}

This work was supported in part by the Automotive Partnership Canada (APC) program supported by the National Science and Engineering Research Council of Canada (NSERC).

\section{REFERENCES}

[1] D. Singh, "Calorimetric Measurement of the Stator Core Losses Caused by Manufacturing," Msc Thesis, Department of Electrical Engineering, Aalto University, Espoo, Finland, 2011.

[2] J. Kwang-Young, P. Chan-Hyuck, and K. Chang-Seop, "Comparison of iron loss at different manufacturing process of actual stator core," in 2013 International Conference on Electrical Machines and Systems (ICEMS), 2013, pp. 523-525.

[3] H. A. Weiss, N. Leuning, S. Steentjes, K. Hameyer, T. Andorfer, S. Jenner, et al., "Influence of shear cutting parameters on the electromagnetic properties of non-oriented electrical steel sheets," Journal of Magnetism and Magnetic Materials, vol. 421, pp. 250$259,1 / 1 / 2017$.

[4] H. Naumoski, B. Riedmüller, A. Minkow, and U. Herr, "Investigation of the influence of different cutting procedures on the global and local magnetic properties of non-oriented electrical steel," Journal of Magnetism and Magnetic Materials, vol. 392, pp. 126-133, 10/15/ 2015.

[5] X. Xiong, S. Hu, K. Hu, and S. Zeng, "Texture and magnetic property evolution of non-oriented $\mathrm{Fe}-\mathrm{Si}$ steel due to mechanical cutting," Journal of Magnetism and Magnetic Materials, vol. 401, pp. 982-990, 3/1/ 2016.

[6] K. Fujisaki, R. Hirayama, T. Kawachi, S. Satou, C. Kaidou, M. Yabumoto, et al., "Motor Core Iron Loss Analysis Evaluating Shrink Fitting and Stamping by Finite-Element Method," IEEE Transactions on Magnetics, vol. 43, pp. 1950-1954, 2007.

[7] A. J. Moses and H. Rahmatizadeh, "Effects of stress on iron loss and flux distribution of an induction motor stator core," IEEE Transactions on Magnetics, vol. 25, pp. 4003-4005, 1989.

[8] A. Daikoku, M. Nakano, S. Yamaguchi, Y. Tani, Y. Toide, H. Arita, et al., "An accurate magnetic field analysis for estimating motor characteristics taking account of stress distribution in the magnetic core," IEEE Transactions on Industry Applications, vol. 42, pp. 668-674, 2006.

[9] N. Takahashi, H. Morimoto, Y. Yunoki, and D. Miyagi, "Effect of shrink fitting and cutting on iron loss of permanent magnet motor," Journal of Magnetism and Magnetic Materials, vol. 320, pp. e925e928, 10// 2008.

[10] K. Yamazaki and A. Aoki, "3-D Electromagnetic Field Analysis Combined with Mechanical Stress Analysis for Interior Permanent Magnet Synchronous Motors," presented at the 20th International Conference on the Computation of Electromagnetic Fields (Compumag 2015), Montreal, Canada, 2015.

[11] J. Zou, J. Li, and Y. Xu, "Research on the Stator Core Loss of the Permanent Magnet Synchronous Motor under Compressive Stress," presented at the 20th International Conference on Soft Magnetic Materials, Kos, Greece, 2011.

[12] N. Takahashi and D. Miyagi, "Effect of stress on iron loss of motor core," in 2011 IEEE International Electric Machines \& Drives Conference (IEMDC), 2011, pp. 469-474.
[13] K.-i. Yamamoto, T. Hamada, and S. Yanase, "Effects of compressive stress normal to the surface of grain-oriented Si-Fe sheets," presented at the $1 \& 2$ Dimensional Magnetic Measurement and Testing, Torino, Italy, 2014.

[14] M. Kawabe, T. Nomiyama, A. Shiozaki, M. Mimura, N. Takahashi, and M. Nakano, "Magnetic Properties of Particular Shape Specimen of Nonoriented Electrical Steel Sheet Under Compressive Stress in Thickness Direction," IEEE Transactions on Magnetics, vol. 48, pp. 3462-3465, 2012.

[15] K.-i. Yamamoto and S. Yanase, "Magnetic Properties of NonOriented Electrical Steels under Compressive Stress Normal to Their Surface," Electrical Review, vol. 87 No. 9b, pp. 97-100, 2011.

[16] D. Miyagi, Y. Aoki, M. Nakano, and N. Takahashi, "Effect of Compressive Stress in Thickness Direction on Iron Losses of Nonoriented Electrical Steel Sheet," IEEE Transactions on Magnetics, vol. 46, pp. 2040-2043, 2010.

[17] K.-i. Yamamoto and S. Yanase, "Magnetic Properties of NonOriented Electrical Steels under Compressive Stress Normal to Their Surface," Electrical Review, vol. 87 No. 9b, pp. 97-100, 2011.

[18] M. Kawabe, T. Nomiyama, A. Shiozaki, M. Mimura, N. Takahashi, and M. Nakano, "Magnetic Properties of Particular Shape Specimen of Nonoriented Electrical Steel Sheet Under Compressive Stress in Thickness Direction," IEEE Transactions on Magnetics, vol. 48, pp. 3462-3465, 2012.

[19] M. F. Ashby, Materials Selection in Mechanical Design, 3rd ed.: Elsevier Butterworth-Heinemann, 2005.

[20] A. Hasenzagl, B. Weiser, and H. Pfützner, "Novel 3-phase excited single sheet tester for rotational magnetization," Journal of Magnetism and Magnetic Materials, vol. 160, pp. 180-182, 1996.

[21] S. Tumanski, Handbook of Magnetic Measurements. Boca Raton: Taylor \& Francis Group, 2011.

[22] E. P. Wohlfarth, Handbook of Ferromagnetic Materials, vol. 2: North-Holland Publishing Company, 1980.

[23] V. Permiakov, L. Dupré, A. Pulnikov, and J. Melkebeek, "Loss separation and parameters for hysteresis modelling under compressive and tensile stresses," Journal of Magnetism and Magnetic Materials, vol. 272-276, Supplement, pp. E553-E554, $5 / / 2004$.

[24] H. Tada, H. Fujimura, and H. Yashiki, "Influence of magnetostriction on hysteresis loss of electrical steel sheet," Journal of Magnetism and Magnetic Materials, vol. 326, pp. 217219, $1 / / 2013$.

[25] G. Bertotti, Hysteresis in Magnetism: For Physicists, Materials Scientists, and Engineers, 1st ed.: Elsevier Science, 1998.

[26] A. Hubert and R. Schäfer, Magnetic Domains: The Analysis of Magnetic Microstructures, 1st ed.: Springer, 1998.

[27] K.-I. Yamamoto, E. Shimomura, K. Yamada, and T. Sasaki, "Effects of external stress on magnetic properties in motor cores," Electrical Engineering in Japan, vol. 123, pp. 15-22, 1998. 